\title{
Fermilab
}

FERMILAB-CONF-06-157-E

soha ${ }^{\circ L W I} 2006^{*}$ writeup

\section{NEW PHENOMENA SEARCHES AT CDF}

\author{
ARON SOHA* \\ University of California at Davis, Department of Physics \\ One Shields Avenue, Davis, California 95616, USA \\ E-mail: soha@fnal.gov
}

\begin{abstract}
We report on recent results from the Collider Detector at Fermilab (CDF) experiment, which is accumulating data from proton-antiproton collisions with $\sqrt{s}=1.96 \mathrm{TeV}$ at Run II of the Fermilab Tevatron. The new phenomena being explored include Higgs, Supersymmetry, and large extra dimensions. We also present the latest results of searches for heavy objects, which would indicate physics beyond the Standard Model.
\end{abstract}

\section{Standard Model Higgs in $h^{0} \rightarrow W W^{(*)}$}

At the Tevatron, the predicted dominant channel for creating Higgs bosons is single neutral $h^{0}$ production. For masses of $m_{\mathrm{Higgs}}>135 \mathrm{GeV} / c^{2}$, the largest decay branching ratio is $h^{0} \rightarrow W W$ (at lower masses, $h^{0} \rightarrow b \bar{b}$ dominates). In the analysis reported here, one or both of $W$ bosons can be off shell, and both are reconstructed in either of the leptonic decays $W \rightarrow e \nu$ or $W \rightarrow \mu \nu$. Prior to a series of event selection requirements, the sample is dominated by Drell-Yan events. After the selection requirements, the azimuthal angle between the two final state leptons is used to separate $h^{0} \rightarrow W W$ signal from the dominant remaining Standard Model (SM) background, which is $W W$ production.

This analysis uses $360 \mathrm{pb}^{-1}$ of CDF Run $\mathrm{II}^{1}$ data to set a $95 \%$ C.L. limit on the cross section times branching ratio, as shown in Figure 1. This new result extends the explored mass range to $110-200 \mathrm{GeV} / c^{2}$, and increases the acceptance beyond that of previous searches. Of the SM Higgs channels being studied at the Tevatron, the $h^{0} \rightarrow W W$ sensitivity is the closest to the corresponding SM prediction, and is about a factor of two from the prediction of a model with a $4^{\text {th }}$ generation of fermions ${ }^{2}$.

$\overline{{ }^{*} \text { On behalf of the cdf collaboration. }}$ 

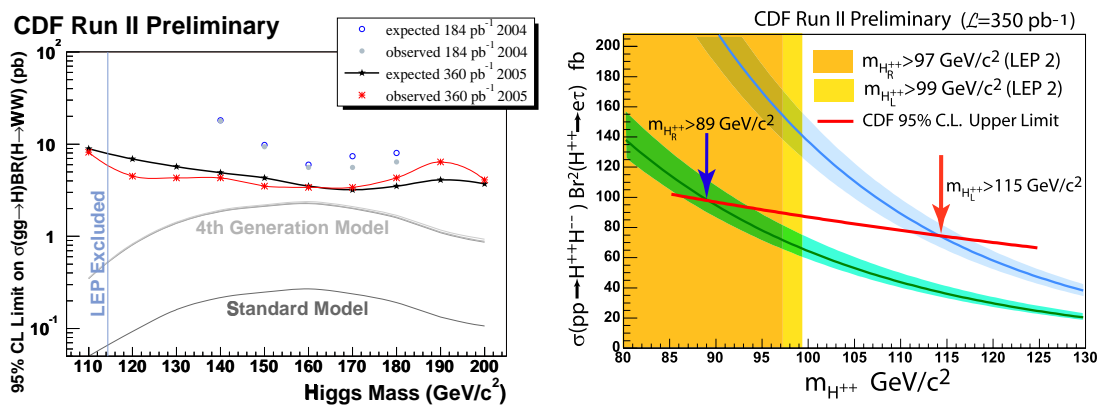

Figure 1. Left: Limit (at 95\% C.L.) on the cross section times branching ratio for $g g \rightarrow$ $h^{0} \rightarrow W W$, including expectations and observations for a new result using $360 \mathrm{pb}^{-1}$ of CDF Run II data. Right: Doubly charged Higgs search results, showing the cross section times branching ratio squared limit, and mass limits for left- and right-handed models.

\section{Search for $\mathrm{H}^{++} \mathrm{H}^{--}$}

We have completed a search for pair produced doubly charged Higgs bosons, which, in certain models ${ }^{3}$, may be as light as $100 \mathrm{GeV} / c^{2}$ and decay primarily to leptons. The production is through $q \bar{q} \rightarrow Z^{0} / \gamma^{*} \rightarrow H^{++} H^{--}$. The lepton-flavor violating decay is theoretically unconstrained, so we utilize the powerful experimental signatures of simultaneous $H^{++} \rightarrow \tau^{+} e^{+}$ and $H^{--} \rightarrow \tau^{-} e^{-}$decays. A requirement of three or four isolated leptons reduces the hadronic (QCD) component of the backgrounds. An additional requirement of $\mathscr{F}_{T}+\Sigma p_{T}$ (leptons) $>190 \mathrm{GeV}$ and a veto against candidate $Z^{0}$ bosons reduce the remaining electroweak backgrounds.

The analysis uses $350 \mathrm{pb}^{-1}$ of CDF Run II data and, with an expectation of 0.25 events from backgrounds, zero events are observed. This gives a $95 \%$ C.L. limit on the cross section times branching ratio squared of $\sigma\left(p \bar{p} \rightarrow H^{++} H^{--}\right) \times B r^{2}\left(H^{++} \rightarrow e \tau\right)<73.5 \mathrm{fb}$, and limits on the mass of $m_{H_{L}^{++}}>115 \mathrm{GeV} / c^{2}$ and $m_{H_{R}^{++}}>89 \mathrm{GeV} / c^{2}$ for left- and right-handed doubly charged Higgs bosons, respectively. Figure 1 shows these results.

\section{Large Extra Dimensions}

The discovery of large extra dimensions would be consistent with a theory of gravitons populating a $4+n$ dimensional bulk, where $n$ is the number of extra dimensions ${ }^{4}$. At the Tevatron, gravitons could be produced directly, in association with a gluon or quark, in $q \bar{q} \rightarrow g G, q g \rightarrow q G$, or $g g \rightarrow g G$. In each case, the signature is an energetic jet and missing transverse energy. This analysis requires jet $E_{T}>150 \mathrm{GeV}$ and $\mathbb{E}_{T}>120 \mathrm{GeV}$. 
The largest SM background is from $Z^{0} \rightarrow \nu \nu+$ jets events, which give $\not_{T}$ due to the neutrinos and is irreducible. There are smaller contributions from $W \rightarrow \ell \nu+$ jets, where there is $\mathbb{E}_{T}$ from the neutrino or a lost lepton, and from QCD processes, where there is $\mathbb{F}_{T}$ from the mis-measurement of jets. The total background expectation, for the $368 \mathrm{pb}^{-1}$ of CDF Run II data considered, is $265 \pm 30$ events. The observation of 263 events is consistent with background and is used to place lower limits on the effective Planck scale, $M_{D}$, of the extra dimensions, and upper limits on the size, $R$, of the extra dimensions, assuming compactification on a torus. The two quantities are related to the Planck mass, $M_{\text {Planck }}$, through the expression $M_{\text {Planck }}^{2} \sim$ $R^{n} M_{D}^{2+n}$. The $95 \%$ C.L. lower limits on $M_{D}$ are $M_{D}>1.16 \mathrm{TeV}, M_{D}>$ $0.98 \mathrm{TeV}, M_{D}>0.90 \mathrm{TeV}, M_{D}>0.85 \mathrm{TeV}$, and $M_{D}>0.83 \mathrm{TeV}$, for $n=$ $2,3,4,5$, and 6 , respectively. The corresponding upper limits on $R$ are $R<0.36 \mathrm{~mm}, R<3.7 \times 10^{-6} \mathrm{~mm}, R<1.1 \times 10^{-8} \mathrm{~mm}, R<3.5 \times 10^{-10} \mathrm{~mm}$, and $R<3.4 \times 10^{-11} \mathrm{~mm}$.

\section{Heavy Objects}

A search for a heavy $Z^{\prime}$ object is carried out by looking for a peak in the di-electron mass, $M_{e e}$, and a distortion in the $\cos \left(\theta^{*}\right)$ distribution ${ }^{5}$. The dominant background is from Drell-Yan production and is estimated using Monte Carlo simulation. For the several models explored, it is found that the data is more consistent with $Z / \gamma^{*}$ plus backgrounds than with $Z / \gamma^{*} / Z^{\prime}$ plus backgrounds, where this notation indicates that a $Z^{\prime}$ would interfere with the $Z / \gamma^{*}$, just as $Z$ and $\gamma^{*}$ interfere in the SM. In $448 \mathrm{pb}^{-1}$ of CDF Run II data, 120 events were observed for $M_{e e}>200 \mathrm{GeV} / c^{2}$, compared to $115_{-19}^{+16}$ events expected from the SM. This is used to set a $95 \%$ C.L. lower limit on the mass of a $Z^{\prime}$ in the sequential model, which is often used for comparisons, of $M_{Z^{\prime}}^{(\mathrm{seq})}>855 \mathrm{GeV} / c^{2}$. The $M_{e e}$ and $\cos \left(\theta^{*}\right)$ distributions are shown in Figure 2.

Another broad approach, open to such models as heavy quarks, extra dimensions, and Supersymmetry, is to perform a signature based search for anomalous dilepton $(e \mu)+\mathrm{X}$ events, where $\mathrm{X}$ can be large $E_{T}$ jets, b-quark jets, a third lepton, large $\mathbb{H}_{T}$, or large event $H_{T}$, where $H_{T}=E_{T}(e)+$ $p_{T}(\mu)+E_{T}($ jets $)+\not_{T}$. Here we apply this strategy to study a heavy quark model with down-type iso-singlet right-handed quarks ${ }^{6}$. We search for an $e \mu$ pair plus two or more jets with $E_{T}>50 \mathrm{GeV}$ and look for any excess in the region $H_{T}>400 \mathrm{GeV}$. With no events seen in $305 \mathrm{pb}^{-1}$ of CDF Run II data, compared to an expectation of $0.802 \pm 0.440$ from the SM, a $90 \%$ C.L. 

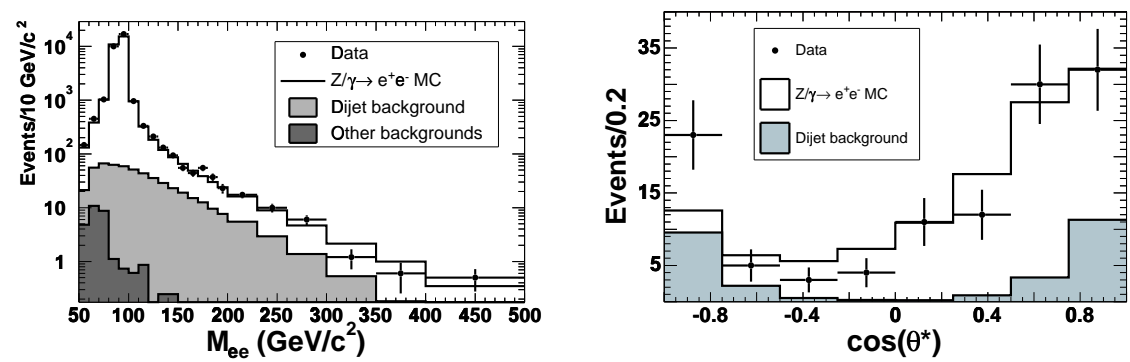

Figure 2. The $M_{e e}$ (left) and $\cos \left(\theta^{*}\right)$ (right) distributions for a $Z^{\prime}$ search. The $\cos \left(\theta^{*}\right)$ distribution includes a requirement of $M_{e e}>200 \mathrm{GeV} / c^{2}$.

limit is set at $4.49 \times \sigma_{Q}$ where $\sigma_{Q}=0.290 \mathrm{pb}$ would be the cross section for a $300 \mathrm{GeV} / c^{2}$ heavy quark.

Finally, we look for heavy objects, which could be heavy quarks, $Z^{\prime}$, or Supersymmetric particles, for example, that decay to $Z^{0}$ bosons. High $p_{T} Z^{0}$ bosons are reconstructed in the $Z^{0} \rightarrow e e$ and $Z^{0} \rightarrow \mu \mu$ channels, with a requirement of $66<M_{\ell \ell}<116 \mathrm{GeV} / c^{2}$. In the future, $\mathrm{W}$ decays and the presence of additional objects such as a photon or b-quark jet will be considered. With $305 \mathrm{pb}^{-1}$ of CDF Run II data, the $Z^{0} p_{T}$ spectrum agrees between data and SM background predictions from Monte Carlo simulation. Limits are obtained on the differential cross section of extra $Z^{0}$ production as a function of $p_{T}$. For the signature of the heavy quark model mentioned above, the $95 \%$ C.L. upper limit on the cross section is $\sigma<0.170 \pm 0.005 \mathrm{pb}$ for a $300 \mathrm{GeV} / c^{2}$ quark.

\section{Search for R-Parity Violating Supersymmetric top}

We search for pair produced Supersymmetric top (stop) quarks at the Tevatron. If R-parity is violated, each stop quark could decay to a tau lepton and b-quark. Our experimental signature is two jets, a lepton or muon from one tau decay, and a hadronic tau decay $\left(\tau_{h}\right)$. Backgrounds from $Z^{0}+$ jets and $\mathrm{QCD}$ are reduced using a requirement of $p_{T}$ (lepton) $+p_{T}\left(\tau_{h}\right)+\mathbb{F}_{T}>85 \mathrm{GeV}$. The $W+$ jets background, where a jet fakes the hadronic tau, is reduced using the transverse mass of the lepton $+\not_{T}$ system. In $322 \mathrm{pb}^{-1}$ of CDF Run II data, 2 events are seen, compared to $2.25_{-0.22}^{+0.46}$ expected from SM sources. This gives a $95 \%$ C.L. lower limit on the stop mass of $m_{\text {stop }}>155 \mathrm{GeV} / c^{2}$. The results are shown in Figure 3 . 


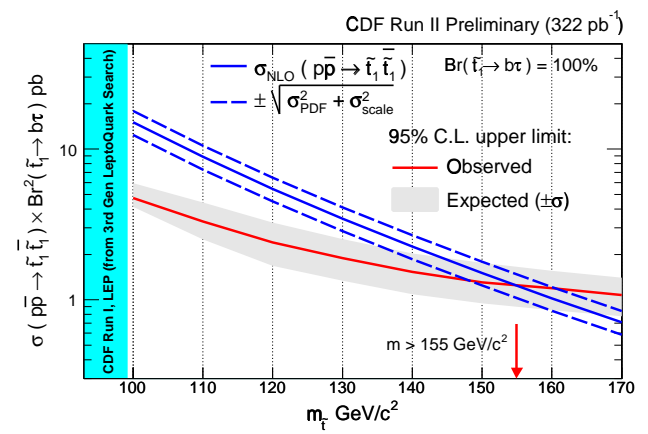

Figure 3. The 95\% C.L. limits for the R-parity violating Supersymmetric top search.

\section{Conclusions}

We have presented a portion of the new phenomena search results available from CDF. In the Higgs sector, the channel $h^{0} \rightarrow W W^{(*)}$ is the most sensitive for higher mass SM Higgs, and limits have also been set for $\mathrm{H}^{++} \mathrm{H}^{--}$. In a search for large extra dimensions, limits have been set on the mass scale and radii. For heavy objects, limits have been set for $Z^{\prime}$, dilepton $+\mathrm{X}$, and high $p_{T} Z^{0}$ production models. Finally, limits have been set for the production and decay of R-parity violating Supersymmetric top quarks. At $\mathrm{CDF}$, both the data sample and potential for discovery continue to grow.

\section{Acknowledgments}

We thank our colleagues within the CDF collaboration, the Lake Louise conference organizers and participants, and the funding agencies for making this work possible.

\section{References}

1. F. Abe et al., Nucl. Instrum. Methods Phys. Res. A271, 387 (1988); D. Amidei et al., Nucl. Instum. Methods Phys. Res. A350, 73 (1994); F. Abe et al., Phys. Rev. D52, 4784 (1995); P. Azzi et al., Nucl. Instrum. Methods Phys. Res. A360, 137 (1995); The CDFII Detector Technical Design Report, FermilabPub-96/390-E.

2. E. Arik, O. Cakir, S. A. Cetin, and S. Sultansoy, Phys. Rev. D66, 033003 (2002).

3. J. F. Gunion et al., Phys. Rev. D40, 1546 (1989).

4. N. Arkani-Hamed, S. Dimopoulos, and G. Dvali, Phys. Lett. B429 (1998).

5. Submitted to Phys. Rev. Lett., hep-ex/0602045 (2006).

6. J. D. Bjorken, S. Pakvasa, and S. F. Tuan, Phys. Rev. D66 053008 (2002). 